\title{
Comparative Effects of Cane Sugar, Honey \& Jaggery on Plasma Glucose Level \& Body Weight of Alloxan Induced Diabetic Rats
}

\author{
${ }^{1}$ Ambreen Anjum, ${ }^{2}$ Hamid Javed Qureshi, ${ }^{3}$ Saima Tabassum, ${ }^{1}$ Wardah Anwar, ${ }^{4}$ Rida Shakil, \\ ${ }^{5}$ Maria Anwar, ${ }^{6}$ Muhammad Hashim Ghouri \\ ${ }^{I}$ Department of Physiology, Al-Aleem Medical College, Lahore \\ ${ }^{2}$ Department of Physiology, Akhtar Saeed Medical College, Lahore \\ ${ }^{3}$ Department of Physiology, Niazi Medical \& Dental College, Sargodha \\ ${ }^{4}$ Pakistan Institute of Medical Sciences, Islamabad \\ ${ }^{5}$ Department of Physiology, Independent Medical College, Faisalabad \\ ${ }^{6}$ Institute of Physiotherapy, Gulab Devi Hospital, Lahore
}

\begin{abstract}
Introduction: Diabetes mellitus is characterized by hyperglycemia with impaired metabolism of carbohydrates, fats, and proteins resulting from insufficiency of secretion or action of insulin. The interplay of genes and environmental factors is believed to stress glucose homeostasis. Simple dietary and lifestyle modifications have a role in prevention and slow progression of the disease. The choice and role of sweeteners (sugars) such as ordinary cane sugar, honey, and jaggery have always been a difficult decision for patients with Type 2 diabetes.

Aims \& Objectives: Evaluation and comparison of the effects of cane sugar, honey, and jaggery on plasma glucose in the sera and body weights of alloxan-induced male Wistar rats with diabetes.

Place and duration of study: The study was conducted at the animal house of Services Institute of Medical Sciences Lahore for a duration of 20 months approximately.

Material \& Methods: In this experimental study 120 male Wistar (albino) rats were randomly allocated into 4 groups of 30 each. After acclimatization, freshly prepared $2 \%$ alloxan monohydrate was administered to all the rats via intraperitoneal injection in a single dose of $120 \mathrm{mg} / \mathrm{kg}$ body weight. Blood sample via tail tipping method was analyzed for fasting serum glucose at 4th post-diabetes induction day. Rodents having $\geq 200 \mathrm{mg} / \mathrm{dl}$ serum glucose were regarded as type 2 diabetes models for further study. All animals had access to usual pelleted diet ad libitum. Diabetic controls in group I were fed with equivalent amount of distilled water. Group II, III and IV rats were fed freshly prepared sweetener containing cane sugar, honey and jaggery solutions respectively in a dose of $1 \mathrm{~g} / \mathrm{kg}$ body weight dissolved in $2 \mathrm{ml}$ distilled water on pre-scheduled time of the day for 28 days via oral syringe-dosing. Final serum glucose was estimated at $29^{\text {th }}$ day of study by terminal blood sampling and final body weights were recorded. Data analysis was performed using SPSS version 20.
\end{abstract}

Results: Group III (honey fed) rats had least hyperglycemia of $34.53 \pm 77.31 \mathrm{mg} / \mathrm{dL}$, followed by group II (cane sugar fed) rats $(79.31 \pm 73.03 \mathrm{mg} / \mathrm{dL})$ then group I diabetic control rats $(88.11 \pm 92.56 \mathrm{mg} / \mathrm{dL})$ and maximal rise in jaggery fed rats with value of $134.50 \mathrm{mg} / \mathrm{dL}$. Significant reduction $(\mathrm{p}=0.049)$ in body weight $(-40.50 \mathrm{~g})$ was noted in group II rats whereas maximum weight gain occurred in group I $(127.57 \pm 72.475)$, than those of group IV rats $(69.13 \pm 92.88)$ followed by II rats $(38.42 \pm 89.43)$.

Conclusion: The result demonstrates that honey in type 2 diabetes mellitus possess antidiabetic properties via minimum hyperglycemic and weight gaining effects than processed cane sugar. As it was presumed, minimally processed jaggery has not shown significant beneficial effects on serum glucose and body weight. Owing to the advantageous effects on metabolic profile, honey may be regarded as a relatively safer sweetener in diabetics.

Key words: Alloxan Induced Diabetes, Hyperglycemia, Type 2 diabetes mellitus, Diabetes Mellitus,Cane sugar,Honey,Jaggery

\section{INTRODUCTION}

$\mathrm{D}$ iabetes mellitus (DM) describes a group of chronic progressive disorders of the metabolic system characterized by high glucose levels, with impaired metabolism of carbohydrates $(\mathrm{CHO})$, fats, and proteins resulting from insufficiency of secretion or action of endogenous insulin. ${ }^{1}$ In this heterogeneous syndrome genes and environment interact, eventually stressing glucose homeostasis. Dietary choices have a decisive role in the expression of genetic susceptibility to diabetes. ${ }^{2}$ Worldwide diabetes epidemic has been associated 
with the way of life-related risk factors such as obesity, sedentary lifestyle, and low-quality dietary choices. $^{3}$

Neuroscientists scientifically established the notion of innate preference of sweet taste for humans since antiquity. Sugars, utilized as sweeteners are an important ingredient of human food but there has been ample debate regarding their contribution to metabolic syndrome and diabetes. ${ }^{4}$ Evidence shows that extensive utilization of sweeteners is robustly related to a variety of interrelated negative effects and development of type $2 \mathrm{DM}$. Reduced insulin sensitivity has been linked with excessive use of common table sugar (sucrose) and different artificial sweeteners. Considerable research has displayed that majority of type 2 DM cases can be prevented through simple dietary and lifestyle modifications. ${ }^{5}$

Nutritional recommendations by Diabetes Associations have emphasized to attain individualized glycemic, blood pressure, and lipimeic control along with achievement and maintenance of body weight together with delay or prevention of diabetic complications. It also persuades people with type $2 \mathrm{DM}$ to abate their energy intake while maintaining a healthy eating pattern to encourage weight loss and improved glycemic control. It has been acknowledged that judicious usage of sweeteners in modest amounts is acceptable especially those which minimally derange metabolic control and body weight. ${ }^{6}$

One of the most exploited sweetening agent by mankind; white odorless crystalline processed table sugar has been linked closely to the mounting epidemic of obesity, DM, metabolic syndrome, dyslipidemia, fatty liver, and various other related negative health effects. ${ }^{7}$ Clinical researchers are continuously struggling to explore other sugar alternatives possessing less harmful impact on overall human health. So, currently, there is a resurgence of scientific interest in natural medicinal sweeteners such as honey, by researchers, the medical community, and even the general public. ${ }^{8}$

A relatively safer traditional sweetening agent, honey offers incredible antiseptic, antioxidant, antimicrobial, antiplatelet, antinociceptive potentials, cariogenic protection, and immune-boosting properties for the goodness of human health. ${ }^{9}$ Besides the dominant sugar content, honey contains the modest amounts of more than 180 organic and inorganic constituents, vitamins, minerals, proteins and amino acids. It also contains trivial amounts of antioxidants, enzymes, organic acids which impart beneficial health effects to this sweetener. ${ }^{10}$

Before the popularity of refined sugar and other sweeteners, jaggery (derived from sugar cane) was considered one of the dominant forms of cane sugar utilization but today it is just a marginal food on the world market, although still significant in some of the producing countries including Pakistan and India (South Asia). ${ }^{11}$ It is a natural mixture of sugar and molasses. If pure clarified sugar cane juice is boiled, what is left is solid jaggery possessing sucrose (65$85 \%$ ), whereas white sugar contains purely sucrose $(99.7 \%){ }^{12}$ This traditional low priced minimally processed sweetener has been claimed to have health and environmental advantages in comparison with refined cane sugar. Although scarcity of high quality scientific research exists, yet few studies point out significant positive health effects of jaggery.

The impact of jaggery and honey on metabolic variables has shown conflicting results. ${ }^{13,14}$ Presumed anti-diabetic effects of these two sweeteners are much complicated and have not been convincingly compared with the ordinary table sugar. On these grounds, the objective of this investigation was to determine and associate the effects of honey, cane sugar plus jaggery on plasma glucose levels and body weights in alloxan-induced male albino diabetic rats with the purpose to identify the safest sweetening agent for patients with diabetes.

\section{MATERIAL AND METHODS}

\section{Study Design and Setting:}

This Experimental Study was conducted at Department of Physiology, Services Institute of Medical Sciences (SIMS), Lahore Pakistan from March 2013 - November 2015.

\section{Ethical Considerations:}

The study protocol and procedures had the approval from the Research Advisory / Evaluation Committee of College of Physicians and Surgeons (CPSP), for Basic Medical Sciences Islamabad. Animal Research: Reporting of In Vitro Experiments (ARRIVE) guidelines were followed for the conduct of research on lab animals.

\section{Animals:}

12-14 weeks old 120 male albino rats of Wistar strain obtained from the National Institute of Health, Islamabad were housed in polypropylene cages at the Animal House of Services Institute of Medical Sciences, Lahore Pakistan. Housing conditions were thermostatically maintained at controlled ambient temperature $26 \pm 2^{\circ} \mathrm{C}$ and an aerated animal room with a 12 hours' dark and light cycle. Commercially available standard pelleted food ad libitum and tap water in clean bottles were fed to the animals.

Rats were selected as per following criteria: 
Inclusion criteria: Healthy, adult male albino rats, weighing 200-300grams.

Exclusion criteria: Rats who were inactive or with some disease (symptomatic illness) or anomaly.

Rats that didn't develop diabetes after receiving alloxan injection.

\section{Randomization:}

Rats were selected through initial purposive sampling and randomly grouped into 4 groups of 30 rats each using a Random Number Table. Animals were placed in their respective cages with mentioned group name and identification tags.

\section{Procedures and Interventions:}

For one week these rats had access to a normal diet before the commencement of the experiment allowing them to be acclimatized. After seven days, rats fed on a normal diet were deprived of food overnight (for about 12 hours) and were weighed, manually restrained, and then anesthetized with chloroform in a glass dome. Freshly prepared 2\% alloxan monohydrate, as a sole dose of $120 \mathrm{mg} / \mathrm{kg}$ body weight of individual rat was administered through intraperitoneal injection to all the rats of group I, II, III, and IV. ${ }^{15}$ Rats with serum glucose $\geq 200 \mathrm{mg} / \mathrm{dl}$ were regarded as alloxan-induced rats with diabetes and used as type 2 diabetes models for further study. Confirmation of DM was established by estimating fasting plasma glucose at $4^{\text {th }}$ postdiabetes induction (about after 96hours) employing a portable glucometer via tail tipping method. ${ }^{16}$ These blood hyperglycemic values by which diabetes in rats was confirmed were recorded as "initial serum glucose" for all diabetic animals in each group respectively. According to the type of feeding/supplementation of sweetener solutions, groups were named as group I Diabetic Control (only distilled water fed), group II (cane sugar), group III (honey) and group IV (jaggery) respectively.

Three different sweetener solutions were prepared daily from day 1 of experimental study to 28th day, freshly by gentle and thorough mixing of the respective sweetener in $2 \mathrm{ml}$ distilled water. Each experimental animal was fed with the respective sweetener solution in a dose $1 \mathrm{~g} / \mathrm{kg}$ body weight on pre-scheduled time of the day for total 28 days of the study period via oral syringe dosing method. Whereas rats in group I, which served as diabetic controls were given equivalent amount of distilled water.

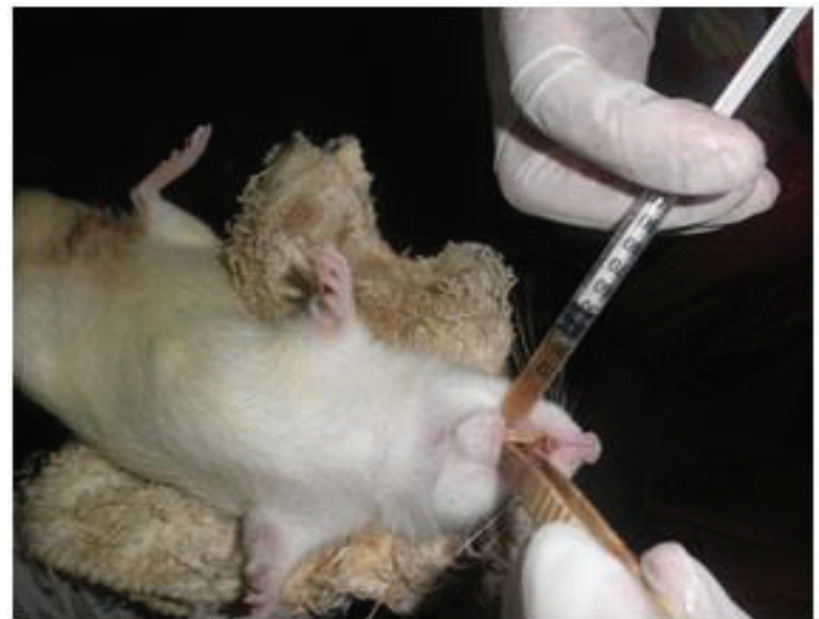

Fig-1: Rat readily drinking sweetener solution $(1 \mathrm{gm} / \mathrm{kg})$ by the oral syringe-dosing

\section{Statistical analysis:}

The data were evaluated using IBM (International Business Machines) Statistical Package for Social Sciences (SPSS), version 20. The normality of data was assessed using the Shapiro-Wilk test. The level of significance was set at $\mathrm{p}<0.05$.

For arithmatical calculations of quantitative variables, mean $\pm \mathrm{SD}$ (Standard deviation) was presented for variables that were distributed normally, and median with IQR (interquartile ratio) were calculated for variables non-normally distributed. For normally distributed variables, parametric tests were used for inferential statistics. One-way ANOVA was applied to observe group mean differences of quantitative variables and Post Hoc Tukey's test was applied to see which group mean differs. For non-normally-distributed variables, non-parametric tests were applied. Kruskal Wallis test was applied to observe the group mean differences of quantitative variables and the Mann Whitney-U test was applied post hoc. Repeated measure analysis of body weight and serum glucose was carried out by using paired samples t-test for normally distributed data and Wilcoxon Matched-Pairs Signed-Ranks Test for non-normally distributed data.

\section{RESULTS}

Effects of sweeteners i.e. cane sugar, honey, and jaggery were evaluated through changing serum glucose levels and body weights in alloxan-induced rats with diabetes. The difference in change of serum glucose after 28 days was highly significant $(p=0.001)$ between the groups. Rise in serum glucose at the termination of study was maximally seen in rats of Group IV (jaggery) as $134.50 \mathrm{mg} / \mathrm{dL}$ (84.25-186.75), followed by Group I (diabetic 
control) rats $(88.11 \pm 92.56 \mathrm{mg} / \mathrm{dL})$ and then in those on cane sugar supplementation $(79.31 \pm 73.03$ $\mathrm{mg} / \mathrm{dL}$ ) while least rise in levels of serum glucose was observed in the rats belonging to Group III (honey) (34.53 $\pm 77.31 \mathrm{mg} / \mathrm{dL})$ (Fig-2).

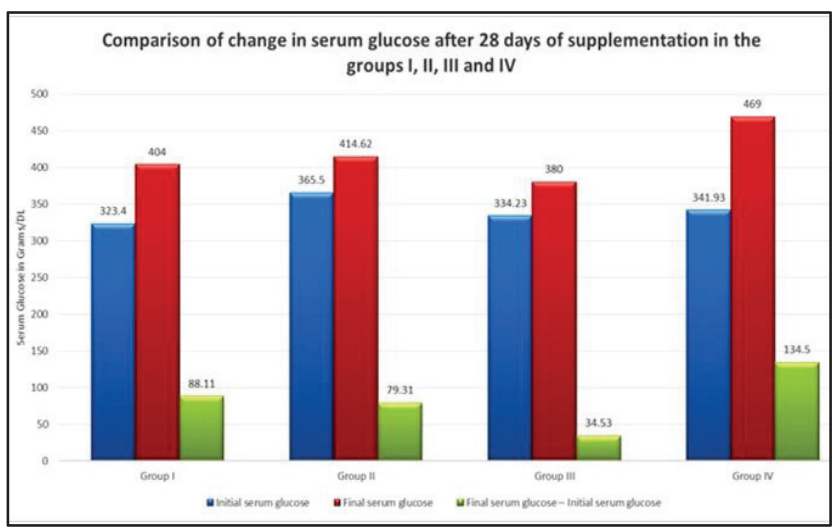

Fig-2: Comparison of change in serum glucose after 28 days of supplementation in the groups, I (Diabetic Control), II (Cane Sugar), III (Honey), and IV (Jaggery).

${ }^{a}$ Values are given as mean \pm SD for normally distributed variables and median (IQR) for non-normally distributed variables.

${ }^{\mathrm{b}} \mathrm{p}$-value is generated by Paired Samples t-test.

${ }^{c}$-value is generated by Wilcoxon Matched-Pairs Signed-Ranks Test.

( $p=0.001$ is considered highly significant and $p<0.05$ is considered significant).

\section{Comparison of change in Body Weight after 28 Days of supplementation in the Groups I, II, III and IV}

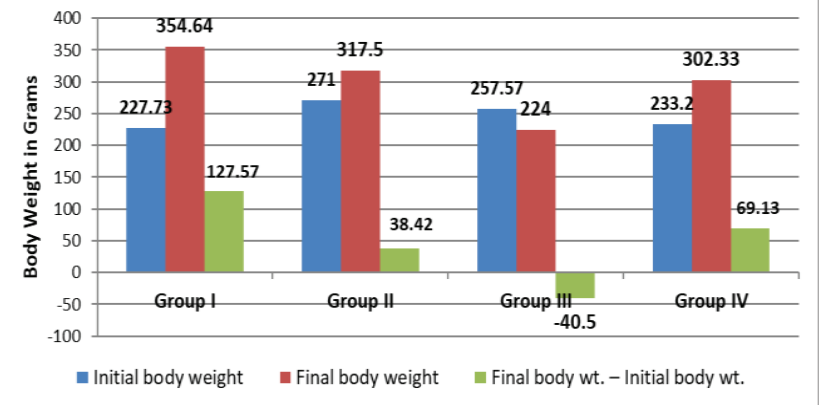

Fig-3: Comparison of Change in Body Weight after 28 Days of supplementation in the Groups, I (Diabetic Control), II (Cane Sugar), III (Honey), and IV (Jaggery).

${ }^{a}$ Values are given as mean \pm SD for normally distributed variables and median (IQR) for non-normally distributed variables.

${ }^{b} p$-value is generated by Paired Samples t-test.

${ }^{c} \mathrm{p}$-value is generated by Wilcoxon Matched-Pairs

Signed-Ranks Test.

$(p=0.001$ is considered highly significant and $p<0.05$ is considered significant).
The initial and final weights of rats significantly $(\mathrm{p}<0.001)$ differed in four groups at the end. Among the total 120 type 2 DM rats, in 28 surviving rats of Group I, at the $29^{\text {th }}$ experimental day, there was highly significant $(p=0.001)$ augmented body weight. \{Final body weight- Initial body weight (grams) $\}$. Similarly, this highly significant increased body weight trend was observed in the 30 rats of Group IV with a p-value of 0.001 , but only a significant increase of weight was observed in 26 surviving rats of Group II with a p-value of 0.040 . On the contrary, bodyweight of rats in Group III was significantly $(\mathrm{p}=0.049)$ reduced with a difference in their Final body weight - Initial body weight as $-40.50 \mathrm{~g}$ (-94.50-27.75) (Fig-3). The control group exhibited a maximum bodyweight gain, followed by the rats on jaggery supplementation and lastly by those given cane sugar. Contrary to this, honey fed rats had shown significantly reduced the body weight.

\section{DISCUSSION}

Our study evaluated the effects of various categories of sweeteners in optimal dose on serum glucose and body weight levels in alloxan induced male diabetic rats. Among these; artificial cane sugar (sucrose), minimally processed jaggery and naturally occurring honey were fed to rats in their respective groups and effects on mentioned parameters were studied compared with the control group.

Minimum rise in levels of serum glucose were observed in the rats belonging to Group III (honey) in our study which are well supported by the demonstration of results by Al-Waili in patients with diabetes when compared with the effects of dextrose and sucrose along with greater elevation of insulin. ${ }^{17}$ Our study has not explored the mechanisms by which honey consumption caused mentioned hypoglycemic effects in type 2 DM rats. Even the current scientific literature could not fully explain the mechanisms by which honey reduces hyperglycemia, weight gain, and other anti-diabetic effects. However, some of the recent studies suggest that the blend of various carbohydrates particularly fructose and oligosaccharides might be the contributory factors. Honey rich in fructose, a naturally occurring sweetener enhances glycemic control much better than sucrose. It has a glycemic index of 19, compared to glucose which has 100 and sucrose (refined sugar) with 60. It suggests that glucose-lowering or antidiabetic properties of honey might be attributed to diminished rate of intestinal absorption, extended gastric emptying duration, and less food intake. ${ }^{18,19}$ 
Significantly differed weight changes among the four groups were observed after 28 days of experimentation. Highest weight gain in diabetic control rats could be due to the polyphagia and increased consumption of the rat chow due to diabetes. This assumption is supported by the evidence of altered balance of hypothalamic neuropeptides, intraneuronal signaling mechanisms in insulin deficiency states. ${ }^{20}$ Results of the current study illustrating positive impact of honey on lowering body weight is consistent with the findings by Chepulis and Starkey in a long term feeding trial who compared the effect of honey against commonly employed sucrose on diabetic rats. ${ }^{21}$

Although it was hypothesized by us that consumption of minimally processed sugar of jaggery would cause relatively less elevation of plasma glucose and minimal weight changes in comparison with the artificial cane sugar but on the contrary significant hyperglycemia and weight gain were observed by the animals in this group. This could be attributed to high glycemic index of jaggery compared with other sweeteners. ${ }^{22}$ In the literature, diverse and contradictory reports are available regarding the metabolic effects of jaggery (and other sugarcane-based minimally processed sugars) in individuals with diabetes. In a closely related remote study, Brekhman and his coresearchers explored the influence of varying amounts of refined white sugar and (organic) brown sugar from common precursor of sugar on certain indicators of $\mathrm{CHO}$ and fat metabolism lasting for about 90 days. The results of this study about the comparative effects of dietary sucrose and muscovado unrefined sugar (very much similar to jaggery) showed minimal derangement in glucose and body weight in the high dose brown sugar fed group compared with sucrose fed rats, suggestive of a potential beneficial nutritive effect of brown sugar (jaggery) on human metabolic health. ${ }^{23}$ Both these contrary studies are quite old and this motivated us to explore and validate the results of experimentation taking honey in account as well. One probable justification for our failure to corroborate the nutritional work of Brekhman might be adoption of unconventional ways of sugar administration as well as different approach for diet formulation.

\section{Limitations and Recommendations:}

The results of present animal based study could be extrapolated to type 2 diabetic human individuals in a meaningful way, if the number of subjects would have been more. Additionally, the promising effects of honey seen in the present experimental study need to be further investigated in well designed, controlled clinical studies to determine whether these can be duplicated in actual clinical situations. Thus, clinical trials with larger patient populations are needed to replicate the results of this study.

Moreover, the chemical characterization (both quantitative and qualitative) of sweeteners used in this research work was not sought out due to technical and financial restrains. This possibly might have given the logical inkling of active constituents of a particular sweetener and its role in type $2 \mathrm{DM}$ in the study subjects.

This study underscores the need for additional studies to elucidate the mechanisms by which honey improves the metabolic control in type $2 \mathrm{DM}$. The promising effects of honey seen in the present experimental study need to be further investigated in well designed, controlled clinical studies on human populations to determine whether these can be duplicated in actual clinical situations.

Furthermore, studies that unravel the true long term effect of minimally processed jaggery in context of DM are recommended.

\section{CONCLUSION}

The findings of this study validate that natural sweetener i.e. (Group III) honey in Type 2 DM has the least weight gaining and hyperglycemic effects than chemically processed cane sugar and jaggery. Whereas the minimally processed Jaggery exhibited considerable hyperglycemia and weight gain as compared to the highly processed table sugar. It suggests the beneficial effects of honey on the overall metabolic profile of subjects with diabetes.

\section{REFERENCES}

1. American Diabetes Association. Classification and diagnosis of diabetes mellitus. Diabetes Care 2017; 40 (Suppl 1):S11-S24.

2. Ericson U, Hindy G, Drake I, Schulz CA, Brunkwall L, Hellstrand S, Almgren P, Orho-Melander M. Dietary and genetic risk scores and incidence of type 2 diabetes. Genes \& Nutrition. 2018; 13:13.

3. Romao I, Roth J. Genetic and environmental interactions in obesity and type 2 diabetes. $\mathrm{J} \mathrm{Am} \mathrm{Diet}$ Assoc. 2008; 108(4):S24-S28.

4. Toop CR, Gentili S. Fructose Beverage Consumption Induces a Metabolic Syndrome Phenotype in the Rat: A Systematic Review and Meta-Analysis. Nutrients 2016; 8, 577.

5. Chong S, Ding D, Byun R, Comino E, Bauman A, Jalaludin B. Lifestyle Changes After a Diagnosis of Type 2 Diabetes. Diabetes Spectr. 2017 Feb; 30(1): 43-50.

6. Evert AB, Boucher JL, Cypress M, Dunbar SA, 
Franz MJ, Mayer-Davis EJ, Neumiller JJ, Nwankwo, Verdi CL, Urbanski P, Yancy WS. Position Statement: Nutrition therapy recommendations for the management of adults with diabetes. Diabetes Care 2014; 36: 3821-42.

7. Laverty AA, Magee L, Monteiro CA, Saxena S, Millett C. Sugar and artificially sweetened beverage consumption and adiposity changes: National longitudinal study. Int J Behav Nutr Phys Act. 2015 Oct 26; 12:137.

8. Bobiş O, Dezmirean DS, Moise AR. Honey and Diabetes: The Importance of Natural Simple Sugars in Diet for Preventing and Treating Different Type of Diabetes. Oxid Med Cell Longev. 2018; 4757893.

9. Meoa SA, Al-Asiri SA, Mahesa AL, Ansari MJ. Role of honey in modern medicine. Saudi Journal of Biological Sciences. 2017 July; Volume 24:5,975-8.

10. Cortés ME, Vigil P, Montenegro G. The medicinal value of honey: A review on its benefits to human health, with a special focus on its effects on glycemic regulation. Cien Inv Agr 2011, 38: 303-17.

11. Walter R. Jaffe. Health Effects of Non-Centrifugal Sugar (NCS): A Review. Sugar Tech 2012; 14:87-94.

12. Waheed $\mathrm{S}$, Ahmad S. Instrumental neutron activation analysis of different products from the sugarcane industry in Pakistan - Part 1: essential elements for nutritional adequacy. AOAC 2008; 2: 392-9.

13. Galvez L, Kwon YI, Genovese MI, Lajolo FM, Shetty K. Antidiabetes and antihypertension potential of commonly consumed carbohydrate sweeteners using in vitro models. Journal of Medicinal Food 2008; 11: 337-48.

14. Okabe T, Toda T, Inafuku M, Wada K, Iwasaki H, $\mathrm{Oku}$ H. Antiatherosclerotic functions of kokuto, Okinawan noncentrifuged cane sugar. Journal of Agriculture and Food Chemistry 2009; 57: 69-75.

15. Jain S, Bhatia G, Barik R, Kumar P, Jain S, Dixit VK. Antidiabetic activity of Paspalum Scrobiculatum Linn in alloxan induced diabetic rats. J Ethnopharmacol. 2010; 127: 325-8.

16. Guidelines for Rodent Blood Withdrawal and Tail Biopsy. The Univeristy of North Carolina at Chapel Hill. 2002.

17. Al-Waili NS. Natural honey lowers plasma glucose, C-reactive protein, homocysteine, and blood lipids in healthy, diabetic, and hyperlipidemic subjects: comparison with dextrose and sucrose. Journal of Medicinal Food. 2004 Apr 1; 7(1):100-7.

18. Erejuwa OO, Sulaiman SA, Wahab MS. Oligosaccharides might contribute to the antidiabetic effect of honey: a review of the literature. Molecules. 2012 Jan; 17(1):248-66.

19. Bobiş O, Dezmirean DS, Moise AR. Honey and Diabetes: The Importance of Natural Simple Sugars in Diet for Preventing and Treating Different Type of Diabetes. Oxid Med Cell Longev. 2018 Feb 4; 4757893.

20. Namkoong C, Kim MS, Jang PG, Han SM, Park HS, Koh EH, Lee WJ, Kim JY, Park IS, Park JY, Lee
KU. Enhanced hypothalamic AMP-activated protein kinase activity contributes to hyperphagia in diabetic rats. Diabetes. 2005; 54:63-8.

21. Chepulis L M, Starkey N. The long-term effects of feeding honey compared with sucrose and a sugarfree diet on weight gain, lipid profiles, and DEXA measurements in rats. Journal of Food Science 2008; 73: $1-7$.

22. Uma P, Hariharan RS, Ramani V, Seshiah V. Glycaemic indices of different sugars. Int. J. Diab. Dev. Ctries. 1987; 7: 78-82.

23. Brekhman I I, Nesterenko I F. Brown sugar and health. Oxford: Pergarnon Press, Oxford, New York, Toronto, Sidney, Paris, Frankfurt. 1983.

\section{The Authors:}

Dr. Ambreen Anjum

Assistant Professor,

Department of Physiology,

Al Aleem Medical College, Lahore.

Prof. Hamid Javed Qureshi

Department of Physiology,

Akhtar Saeed Medical College, Lahore.

Dr. Saima Tabassum

Assistant Professor,

Department of Physiology,

Niazi Medical \& Dental College, (Sargodha)

Dr. Wardah Anwar

Assistant Professor

Department of Physiology,

Al Aleem Medical College, Lahore.

Dr. Rida Shakil

Medical Officer,

Pakistan Institute of Medical Sciences, Islamabad.

Dr. Maria Anwar

Assistant professor,

Department of Physiology,

Independent Medical College, Faisalabad.

Muhammad Hashim Ghouri

IASTM Institute of Physiotherapy,

Gulab Devi Hospital, Lahore.

President, Pakistan Research and Welfare Society

\section{Corresponding Author:}

Dr. Ambreen Anjum

Assistant Professor, Department of Physiology,

Al Aleem Medical College, Lahore.

E-mail: draanjum28@yahoo.com 